\title{
Study on Linkage Mechanism of Enrollment, Cultivation and
}

\section{Employment of Local Universities}

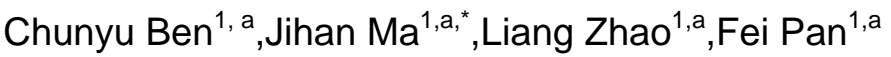 \\ ${ }^{1}$ Examination Institute of Jilin Province, Changchun 130033, China; \\ jeffy2010@126.com
}

Keywords: Enrollment, Cultivation, Employment, University

\begin{abstract}
The era of big data, provide a good opportunity for the development of higher education, but also new challenges. Colleges and universities must adapt to the requirements of the times, the $r$ eform of the old model, continuous innovation, set up to meet the requirements of the times of mod ern management mode and management system. By establishing database, design the related content, to under the background of big data analysis of linkage mechanism based on the recruit students - culture - employment decision-making and management system for research and exploration. On the basis of existing orientation registration system and planning system, continuously integrating and expanding its related functions, forming a complete admissionsculture - job analysis decision management system.
\end{abstract}

\section{Preface}

At present, some scholars on enrollment, training, employment of the linkage relationship has been studied, some colleges and universities have also tried to recruit students, training, employment link age reform. Mao song Wang outlined the undergraduate colleges and universities to meet the needs of society oriented recruitment, training, employment integration project, operation mechanism as $\mathrm{f}$ our 'three', that is, to establish three kinds of concepts, the formation of three institutions, to realize $t$ he three docking, to build three linkage mechanism. Xie Aijun, Wang Hui, Peng Xilin proposed the new ways to explore the employment of graduates in colleges and universities must be from the recr uitment, training, employment relation, in educational practice to grasp its internal rules, so as to est ablish the recruitment, training, employment Trinity linkage mechanism, to expand the space for em ployment of graduates. Huang Zhaoxin pointed out that with Scientific Outlook on Development to build the college enrollment, training and employment system reform. Although the present to discu ss the establishment of recruitment, training and employment linkage mechanism for the number,tha $\mathrm{t}$ in the context of large data research and practice analysis management system based on linkage $\mathrm{m}$ echanism is still blank. The era of big data, provide a good opportunity for the development of high er education, but also new challenges. Colleges and universities must adapt to the requirements of $\mathrm{t}$ he times, the reform of the old model, continuous innovation, set up to meet the requirements of the times of modern management mode and management system. This paper is based on the analysis of the connotation and characteristics of large data, for local colleges and universities based on enroll ment training employment linkage mechanism analysis and decision system, management, research the necessity of research, in the fusion of recruitment, training, employment and so on massive data based on , reasonable design system and the final completion of the development and debugging of the system. 


\section{Research target}

With the continuous reform and development of higher education, the practice of increasing diversit $\mathrm{y}$, democracy, increasingly fierce competition between high quality students in colleges and univers ities, graduates employment situation has become increasingly serious. Universities have appeared different degree of teacher shortage phenomenon, academic and professional structure contradiction s increasingly prominent, some schools according to the situation of the talent needs of society and not blindly expanding enrollment, the happening of the structural unemployment resulting in colleg e students' employment. In addition, colleges and universities in talents, often in the college entranc e examination scores as the only measure; Recruitment, training and employment, the communicati on between the data sharing mechanism has not yet formed. These problems directly affect the colle ge's educational level and quality of personnel training, caused huge pressure to graduate employme nt. Therefore, to study how to set up the mechanism of recruitment, training, employment, sharing i nformation resources, how to achieve maximum development at a minimum cost, how to make the colleges and universities in the recruitment, training, employment work every year to make the right decisions, has become a new topic to be studied in the colleges and universities.

Based on enrolment in local colleges employment linkage mechanism analysis of the decision-maki ng mechanism research, should achieve the following goals:

First, with the enrollment system, the new registration system, enrollment plan management system, school educational administration management system, employment system and data statistic mana gement system, etc, to achieve full data information sharing, making further improve the data proce ssing of the automation and intelligent level, higher operating efficiency;

Second, use the scientific method, using decision model to analyze huge amounts of data, for the re cruitment, training, provides the reference for the prediction and decision of employment;

Third, reflect the requirements of the development of information technology, the use of computer network technology recruitment, training, publication and exchange of information on employment, expanding the use of the system function.

\section{Solution}

Taking a provincial university as an example, Since 2002 began to use online enrollment mode, After more than ten years of Enrollment, We have accumulated a wealth of enrollment database resources, Student Employment Data Administration Management System over the years and stored in the student information system to collect actual employment has constituted a complete set of students into the school, school data, leave school

Through the application of linkage mechanism, the data warehouse and data mining technology is applied to college enrollment, student management, to establish a based on admission - Training employment linkage mechanism with intelligent management decision analysis system and extract the implicit unknown and valuable information from the massive historical data, scientific guidance to the recruitment, training, employment related work has an important theoretical and practical significance. According to the statistics of the historical data, data mining, extraction of recruit students, training, graduate, employment information, achieve the following expected effect:

(1) to ensure open, fair and transparent admission

College entrance examination from the scene registration to online registration, voluntary reporting, with the continuous development and progress of the national college entrance examination policy, on the work of college admissions information requirements are also increasingly strict, the admission of the principle, the enrollment method, admission information released with more 
specific provisions and requirements. Through the system, the timely release of relevant information to the website for the candidates query.

(2) to meet the needs of complex enrollment data processing

In recent years, most colleges and universities have adopted the "national college admission system" developed by the Ministry of education, which has been developed by the Ministry of education. The admission system will generate a large number of redundant data, and the different provinces of the enrollment data, the definition of the format is not exactly the same. So we need to organize a large number of admission data, statistics report and many other issues, has brought great pressure to the work of each school admissions staff, spend a lot of energy to deal with these data. Therefore, the scientific setting of enrollment data processing process, can greatly improve work efficiency, meet the needs of enrollment data processing complex.

(3) simplify the registration of new students to work

Part of the admission decision analysis processing system, orientation of the registration system will be implemented to fill in personal information, but you can also apply for transfer of registered permanent residence and green channel report, teaching materials, clothing and daily necessities reservation, ahead of the completion of part of the report procedure. Personal information input, goods scheduled, book materials, account migration, loan application a series report process forward, thereby greatly reducing the live report of waiting time, and at the same time, the relevant functional departments more convenient more intuitive to see the new report dynamic, easy data query and statistics. The new registration system research and development, has laid a good foundation for students to advance understanding of the school, as soon as possible into the campus. Simplify the registration procedures, for students and parents to provide the convenient check-in service at the same time, the school also facilitate ahead of time to master the new dynamic report, the relevant functional departments can be convenient for data query and statistical work.

(4) to provide reference for the professional construction and student training

By students to apply for voluntary data analysis to summarize various professional disciplines enrollment changes, convenient for the school to determine what professional needs to be further strengthen construction, step up publicity efforts to or focused on development of what professional, as school brand to attract outstanding students; at the same time, you can according to the interest of freshmen, the hobby, the special skill and other information for teaching, for cultivating the students' individual.

(5)Providing the reference for the school recruitment of students admissions decisionsand plans.

Through the collection, summary and analysis the school admissions number for enrollment, each a dmissions professional plan and actual registration number, various professional teachers, such as e mployment history data. System extract the important information in data. It will provide data sup port and analysis basis for university enrollment work deployment and decision for the future.

(6) Make admissions,foster,employment administration become the closed loop.

Break the strip division of admissions,foster,employment,build the linkage mechanism of those.Ma ke full use of decision management system and scientific analysis of the related data,to product inf ormation of virtuous cycle of admissions, foster,employment.Form the source of the admissions,reg ulating specialty structure,deepening educational reform,improving the quality of training.Making more innovation on employment and improving the employment system.

\section{Conclusion}

This topic research combined survey method, empirical study, qualitative analysis, experience summary method, exploratory research methods. through the research for the local colleges and 
universities of beneficial experience in decision-making and management system, and the recruitment, training, employment of massive amounts of data analysis summary. Exploring the decision-making management of the necessity and feasibility of system development ,at the same time, establishing database, design the related content, to under the background of big data analysis of linkage mechanism based on the recruit students - culture - employment decision-making and management system for research and exploration. On the basis of existing orientation registration system and planning system, continuously integrating and expanding its related functions, forming a complete admissions- culture - job analysis decision management system。 Through the data pretreatment, data conversion, data processing, data statistics, etc ,it will provide accurate digital word basis and useful reference for the local colleges and universities in the future enrollment management, personnel training and employment management.

\section{References}

[1] Huangzhao Xin. Scientific View of Development and Construction of Admission to Employment body system [J]. Higher Education Of Engineering, 2006, (1).

[2]Maotao Wang. Undergraduate needs of the community-oriented recruitment, training, operation mechanism to explore employment integration of Higher Education in Agriculture, 2009, (2).

[3]Shiqing Chen,Shuolai Bao.Local Universities "Admissions·Employment·Training" Construct Integration work mechanism[J].Anqing Normal University Academic Journal(Social Science Edition),2008(12).

[4]Yu Zheng. To promote the employment system of the employment system based on the social needs[J].Fuyang Normal University Academic Journal(Social Science Edition),2008(2).

[5]Aijun Xie,Hui Wang,Xilin Peng. Thinking to establish an efficient mechanism of Admission to Employment and broadening the employment space. Higher Agricultural Education,2009,(2)82-84

Corresponding author:Jihan Ma(1968-),E-mail:jeffy2010@126.com 